The results obtained by the use of the various brands of serum are shown below in a tabular statement. Flexner's serum was employed in 39 cases altogether, with a mortality of 9 , or 23 per cent.; in 27 of the 39 cases it was the only serum used, with a mortality of 6 , or 22 per cent. ; the 12 other cases in which other serums were given as well showed a mortality of 3 , or 25 per cent. The other serums were given in much fewer cases. The mortality percentage of the 14 cases that received Burroughs Wellcome and Co.'s brand (alone or combined with other serums) was slightly lower than that of the cases treated with Flexner's serum; and the 16 cases treated by Gordon's various serums alone showed an even lower mortality,

Table showing Results of Treatment by Different Serums.

\begin{tabular}{|c|c|c|c|}
\hline- & Cases & Deaths. & Recoveries. \\
\hline \multirow[t]{2}{*}{$\begin{array}{c}\text { Flexper's serum alone } \ldots \begin{array}{ccc}\ldots & \ldots & \ldots\end{array} \\
\text { Combined with other serums } \\
\ldots\end{array}$} & $\begin{array}{l}27 \\
12\end{array}$ & $\begin{array}{l}\text { Per cent. } \\
6 \text { or } 22 \cdot 3 \\
3,25\end{array}$ & $\begin{array}{l}\text { Per cent. } \\
21 \text { or } 77 \cdot 7 \\
9,, 75\end{array}$ \\
\hline & 39 & 9 or 23 & 30 or 77 \\
\hline \multirow[t]{2}{*}{$\begin{array}{ccccc}\text { Gordon's serums alone } & \ldots & \ldots & \ldots & \ldots \\
\text { Combined with other serums } & \ldots & \ldots\end{array}$} & $\begin{array}{r}16 \\
6 \\
\end{array}$ & $\begin{array}{l}3 \text { or } 18 \cdot 7 \\
3,50\end{array}$ & $\begin{array}{c}13 \text { or } 81 \cdot 3 \\
3,50\end{array}$ \\
\hline & 22 & 6 or $27 \cdot 3$ & 16 or $72 \cdot 7$ \\
\hline \multirow[t]{2}{*}{ 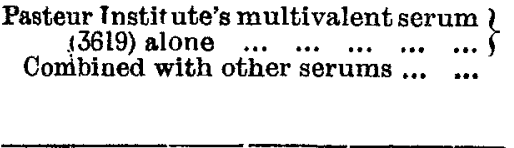 } & $\begin{array}{l}9 \\
5\end{array}$ & $\begin{array}{l}4 \text { or } 44 \cdot 5 \\
1,20 \\
\end{array}$ & $\begin{array}{l}5 \text { or } 55 \cdot 5 \\
4,80 \\
\end{array}$ \\
\hline & 14 & 5 or $35 \cdot 7$ & 9 or $64 \cdot 3$ \\
\hline \multirow[t]{2}{*}{ 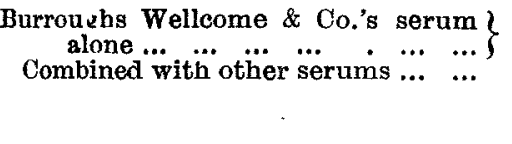 } & $\begin{array}{l}6 \\
8\end{array}$ & $\begin{array}{l}2 \text { or } 33 \cdot 3 \\
1, \quad 12 \cdot 5 \\
\end{array}$ & $\begin{array}{l}4 \text { or } 66 \cdot 7 \\
7,, 87 \cdot 5 \\
\end{array}$ \\
\hline & 14 & 3 or $21 \cdot 4$ & 11 or $78 \cdot 5$ \\
\hline \multirow[t]{2}{*}{$\begin{array}{l}\text { Mulford's serum alone } \ldots \\
\text { Combined with other serums } \ldots\end{array}$} & $\begin{array}{r}10 \\
2\end{array}$ & $\begin{array}{l}5 \text { or } 50 \\
1\end{array}$ & $\begin{array}{l}5 \text { or } 50 \\
1\end{array}$ \\
\hline & 12 & 6 & 6 \\
\hline 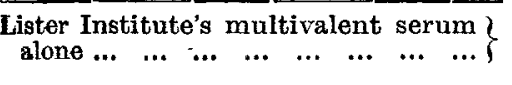 & 11 & 6 or 54.5 & 5 or 45.5 \\
\hline
\end{tabular}

The number of occasions on which serum was given intrathecally varied in the 95 cases from 1 to 8 , according to the duration of the symptoms. In some instances the improvement and fall of temperature after the injection of serum were dramatic.

Sixteen cases received one dose only, and 7 proved fatal, 6 being very acute or fulminating cases; 25 received two doses of serum (8 deaths); 22 cases three doses (7 deaths); 8 cases four doses ( 1 death); 11 cases five doses (4 deaths) 5 cases six doses ( 2 deaths); 4 cases seven doses ( 1 death); and 4 cases eight doses (no death). In many cases lumbar puncture was performed more often than serum was injected; in 1 chronic case in whicb serum was given six times, lumbar puncture was performed on 20 occasions.

Serum rashes, erythematous or urticarial, were mentioned in 22 , or 23 per cent., of the 95 cases treated by serum, but may have been more frequent. The rashes and articular manifestations were practically confined to cases treated with Flexner's and Burroughs Wellcome and Co.'s brands.

Out of the 22 cases 21 received either Flexner's or Burroughs Wellcome and Co.'s serum; 12 had Flexner's serum alone, 2 Flexner's and Gordon's serum, 1 Flexner's and the Pasteur Institute's multivalen't serums, 2 Burroughs Wellcome and Co.'s serum alone, 1 Burroughs Wellcome and Co.'s and Gordon's serums, and 3 Flexner's and Burroughs Wellcome and Co.'s serum (only 4 cases had this combination). The remaining case had 70 c.c. of Gordon's serum, and did not have any manifestations of serum disease except the rash. Ont of the 95 cases treated with serum, 41 had Flexner's or Burroughs Wellcome and Co.'s serum, and among them rashes occurred in 21 , or 51 per cent.; whereas among the remaining 54 cases treated by serum there was a rash in one case only. Six of the 22 cases with rashes also had serum hypodermically. The average amount of serum received by the cases with rashes was 65 c.c.; and the average amount received by 14 other cases which had Flexner's or Burroughs Wellcome and Co.'s serum and recovered was 70 c.c., or practically the same.

On an average the rash appeared ten davs after the first injection of serum. In a case at Haslar which received one injection of 20 c.c. of Flexner's serum in a relapse seven weeks after the primary attack in Egypt, there was within a few hours an "immediate" serum rash. The patient stated that nine lumbar punctures had been performed in the primary attack, but there is no official information as to the administration of serum.

Of the 22 cases none proved fatal, but as the serum rash occurs after an interval of about ten days, the fulminating and acute cases are obviously excluded, and this observation has no prognostic value. Six cases had both herpes and a serum rash ; 9 cases an initial rash and a serum rash; and 3 cases an initial rash, herpes and a serum rash (in one case on two occasions); presumably in these instances the skin was especitlly prone to react.

In some cases pains in the joints without effusion accom. panied the serum rash, and in two instances effusion into the joints and a ternperature of $102^{\circ}$ coincided with the rash. In 2 cases at Chatham temporary deafness occurred at the same time as a serum rash and arthralgia, and was regarded by Temporary Surgeon A. C. McAllister as a manifestation of serum disease.

Grave accidents due to intrathecal injection of antimeningococcic serum were very rare.

In one case lumbar puncture was done directly after admission to the Royal Naval Hospital, Plymouth, and 50 c.c. of turbid fluid containing meningococei spurted out 15 c.c. of Gordon's multivalent serum was given intrathecally and the same quantity hypodermically. Respiration stopped at once. The necropsy showed fibrinous adhesions and purulent streaks over the cerebral sulci, with but little exudation at the base of the brain. Both lateral ventricles were distended with blood-stained serum. The purulent exudate over the hemispheres suggested that the illness had last a week. In another case at Plymouth lumbar puncture on the third day of the disease gave exit to 30 c.c. of turbid fluid, and 15 c.c. of Gordon's multivalent serum were injected intrathecally; five hours later respiration stopped before the pulse. The necropsy showed fibrinopurulent exudate over the base of the brain and much purulent exudate over the base of the brain and much blood-stained fluid in both ventricles, cedema of the cerebellum pressing on the pons, dilatation of the right side of the heart, and recent and old endocarditis of the mitral valve. Although death did not occur until five hours after the injection of serum, this case resembles the first in the presence of blood-stained fiuid in the lateral ventricles of the brain. In a third case death occurred suddenly seven hours after injection of serum and the necropsy showed distension of the lateral ventricles and acute congestion and œdema of the cerebellum pressing on the pons.

In a man who had had six lumbar punctures the site of the punctures became inflamed and death rapidly followed. at the necropsy it was found that the lateral ventricles were greatly distended with pus swarming with streptococci.

In 2 cases lumbar puncture gave rise repeatedly to severe headache, which was partially relieved when serum was injected intrathecally.

\section{FOUR CASES OF HEMIPLEGIA CAUSED BY EMBOLISM FOLLOWING GUNSHOT WOUNDS OF THE CAROTID ARTERIES.}

By L. COLledge, M.B. Cantab., F.R.C.S. Eng., CAPTAIN, R.A.M.C. (T.F.); AND

JOHN SHAW DUNN, M.D., CH.B. GL.ASG., XIEUTENANT, R.A.M.c. (T.C.)

A SERIES of 14 cases has recently been reported by Sir George Makins ${ }^{2}$ in which symptoms of cerebral embolism followed gunshot injury of the carotid arteries. Of these cases only one ended fatally, and in it a post-mortem examination was not available, so that a complete anatomical account of the conditions could not be obtained. Four similar cases, presenting the remarkable clinical picture of a wound in the neck accompanied by hemiplegia and loss of consciousness, and all ending fatally, have come under observation in a casualty clearing station. As a necropsy was performed in every case they appear worthy of record.

CASE 1.-In this case there was a small wound on the left side of the neck, below and behind the angle of the jaw; the exit wound was also on the left side, lower down, about 13 inches from the middle line behind. An extensive diffuse swelling appeared behind the angle of the jaw; this 
swelling did not pulsate, nor was there any bruit. The patient was unconscious and stertorous, and there was hemiplegia affecting the face, arm, and leg on the right
side. The patient died about 26 hours after being wounded.

On dissection of the left sicle of the neck there was noted a localised swelling, with hæmorrhage, on the walls of the external and internal carotid arteries, just above the bifurcation of the common trunk. Two small wounds, with ragged edges, were present, one on the front of the external and the other on the back of the internal artery; these appeared to be sealed up by firm red thrombus which was entangled in projecting sbreds of the adventitial tunics, and the thrombus on the back of the inner vessel exhibited a firm pealike projection backwards. A probe which was readily passed, with light pressure, in at one opening and out at The vessels were removed unopened, the internal carotid being cut across half an inch from the base of the skull. Its lumen was patent at that level, but was filled by thrombus to a point just below. When fluid was poured into the common carotid artery it was observed to make its exit by the external artery, none escaping by the wounds. The lumen of the left internal carotid artery was observed to be patent also at the point where it was cut in removing the brain. The left midale cerebral vessel was normal to a point just beyond the origin of its perforating branches, but was there distended and completely blocked by a red adherent thrombus. The Bacillus perfringens was present in this clot in considerable numbers. There was a wellmarked condition of early softening of the left hemisphere of the brain, excepting the occipital and frontal lobes. The basal nuclei were normal.

CAse 2.-In the second case there were multiple small bomb wounds, one of which was on the right side of the neck posteriorly. There was a similar non-pulsatile swelling of the neck, and the symptoms produced were similar to those in the first case. The patient died, hemiplegic on the left side, about 18 hours after being hit.

On post-mortem examination much effused blood was present in the tissues of the right side of the neck. The missile, a small fragment of bomb-casing, was seen lying under the right sterno-thyroid muscle, having penetrated the right lobe of the thyroid gland. There was a false aneurysm, about an inch in diameter, on the deep internal aspect of the right common carotid artery; its wall was formed of firm fibrin mixed with blood corpuscles. On slitting up the vessel after removal, a linear wound, $\frac{3}{8}$ inch in length, was seen on its posterior and inner wall about $1 \frac{1}{2}$ inches below the bifurcation. A firm cylindrical thrombus was adherent to the upper end of this wound and extended upwards for a distance of 1 inches, lying free in the lumen and only partially filling it. The external and in ternal carotids were free of thrombus as far as they could be traced in the neck, and the latter was patent also at the point where it was cut inside the skull in removing the brain. The right Sylvian artery was completely occluded by firm red thrombus just bevond the origin of its perforating branches. The right hemisphere of the brain showed well-marked early softening, with the exception

FIG. 1.

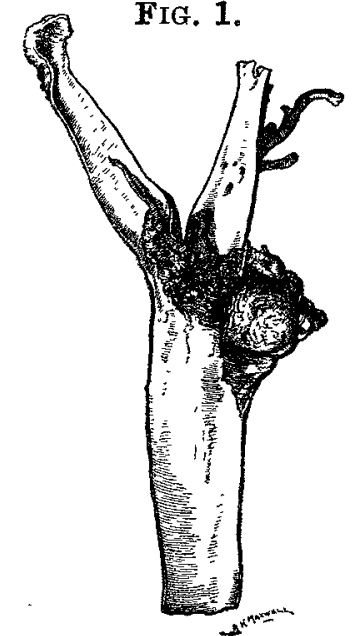

The carotid arteries from Case 3, showing the false aneurysm and the in the wall of the external carotid is quite thrombi inside the
vessels. The perforation of the frontal and occipital poles. The grey matter was swollen, red, and cedematous and had a blurred margin, and contrasted with the normal left side. The basal nuclei were intact.

CASE 3.-This case was admitted within six hours of being wounded with the same symptoms of stertor and hemiplegia. The patient had been shot through the neck from side to side. On the right side there was a small wound near the posterior border of the sterno-mastoid muscle, about midway between its attachments. Arterial bloou escaped freely from this wound when the dressing was removed. The right common carotid was therefore ligatured just above the omo-hyoid; the hæmorrhage was thereby arrested. The patient died without any alteration in the symptoms within 15 hours of being wounded.

In this case a false aneurysm about the size of a walnut was found on the back of the right external carotid artery just above the bifurcation of the main trunk. (Fig. 1.) A small perforation about $\frac{1}{8}$ inch in diameter was present in the posterior wall of the vessel at that level. The edges of this wound were ragged and bruised, and the opening was crossed by some residual strands of adventitia with clot adherent to them. The posterior wall of the internal carotid artery was much bruised at a slightly lower level, but was not perforated. Fairly firm greyish-pink thrombus was adherent on the walls of the common carotid artery at its bifurcation, and extended $\frac{1}{2}$ inch upwards in the lumen of the internal and $1 \frac{1}{2}$ inches in that of the external vessel, being firmly attached over the bruised areas of intima. The external carotid was the only vessel completely occluded by thrombus. Emboli were present in several branches of this vessel in the neck; the lumen of the internal carotid was free of clot from just above the injury on its wall to its termination inside the skull. The right middle cerebral vessel was normal at its origin, but all the larger branches in the Sylvian fissure were filled by portions of thrombus of the same consistence as that seen in the large vessels in the neck. The island of Reil and the motor area of the right cerebral hemisphere were softened and much congested, while the basal nuclei and the poles of the hemisphere remained normal. A few streptococci were present in films from the thrombi, and a few colonies were obtained on cultivation.

CASE 4. - This case was ad mitted about six hours after being wounded, already in the same condition, stertorous, and with hemiplegia affecting the face, arm, and leg on the right side. There was a wound on the left side of the neck, about $2 \frac{1}{2}$ inches above the clavicle. This patient survived for about 72 hours after being wounded.

Post mortem the left common carotid artery was found to be completely severed and the ends, filled with thrombus, were retracted so as to leave a gap half an inch in length. (Fig. 2.) The ends of the vessel were much bruised and some laceration was present for half an inch upwards on the walls of the upper segment. The thrombus in the lower segment was traced downwards to within an inch of the aorta; that in the upper extended up to the bifurcation of the common carotid, and thence for a considerable distance into the external carotid and its branches and for half an inch only into the internal carotid. The lumen of the latter vessel was patent above that level. On the base of the brain the left Sylvian artery was plugged by firm red thrombus beyond the origins of the perforating branches. This had given rise to cerebral softening, as in the previous cases.

In each of these cases the hemiplegia and cerebral disturbance were caused by occlusion of the middle cerebral artery or its branches. In all four there was thrombus present in the carotid arteries in the neck, consequent on damage of the arterial walls, and in each there, were several inches of the internal carotid artery patent between that thrombus and the portion blocking the cerebral vessel. It is consequently a justifiable conclusion that the blood continued to flow for a time, at least, in the wounded vessels, and that the occlusion of the cerebral arteries occurred by embolism. Similar emboli were found in one instance in the branches of the external carotid also.

The damage to the large cervical arteries was in every case considerable. In three there was actual perforation of the wall; in the fourth the trunk was found completely severed, but this, no doubt, occurred secondarily by sloughing, after the vessel was fully thrombosed. It is remarkable that hæmorrhage was so slight in these cases; in only one of them was ligature nesessary.

Sir George Makins draws attention to the tardy development and partial nature of the paresis as outstanding 
features in his series, and contrasts this with the prompt and complete hemiplegia, followed by a fatal issue in 24 to 36 hours, whieh may occur after ligature of the common carotid artery. In the four cases related, however, the early appearance and completeness of the hemiplegia and the rapidly fatal issue resemble the effects of ligature of the common carotid. It is natural that such cases, only surviving from 15 to 72 hours, would not reach a base hospital.

We are indebted to the Medical Research Committee for permission to make use of the two drawings by Mr. A. K. Maxwell.

\section{ON THE PRESENCE OF AN ACCESSORY FOOD FACTOR IN THE NASAL SECRETION}

AND ITS ACTION ON THE GROWTH OF THE MENINGOCOCCUS AND OTHER PATHOGENIC BACTERIA. (Preliminary Paper.)

BY CRESSWELL SHEARER, M.D., F.R.S., MIUTTARY HOSPITAL, DEYONPORT.

(Report to the Medical Research Committee.)

\section{Introduction.}

IN dealing with numbers of cerebro-spinal fever contacts during the recent epidemic it was noticed that the growth of the meningococcus was in some instances strongly influenced by the amount of nasal secretion transferred by the swab to the surface of the culture medium. It was found from experiment that if a trace of dilute "sterile nasal secretion was added to the surface of the culture medium, it brought about a rapid growth of the meningococcus when this medium was subsequently inoculated with this germ. The growth is always many times the amount obtained on the same medium, on planting out a similar quantity of culture, in the absence of nasal secretion. ${ }^{x}$

The action of sterile dilute nasal secretion in accelerating the growth of the meningococcus would seem to be entirely in the nature of an accessory food body or vitamine, as alone this secretion is quite incapable of supporting or stimulating the growth of this germ. This conclusion is supported by its peculiar properties. It is soluble in water, slightly soluble in alcohol, and almost insoluble in ether. ${ }^{2}$ It has great heat-resisting capacity, withstanding autoclaving at $120^{\circ} \mathrm{O}$. and boiling in strong hydrochloric acid for 12 hours without losing strength. On filtration it always undergoes considerable adsorption, even when passed through an ordinary filter-paper, and it almost entirely disappears from any fluid when this is passed through a Doulton filter candle. All chemical manipulation, drying, precipitation, \&c., appreciably weaken its power of stimulating the growth of the meningococcus.

In addition to the meningococcus and its allies, it has been shown to accelerate the growth of the pneumococcus, B. typhosus, B. coli communis, various fæcal streptococci, and a number of throat bacteria. It is highly probable that it will accelerate the growth of all pathogenic bacteria when added in sterile fashion to any suitable culture material, whether solid or fluid. 3

\section{Mode of Collection of Nasal Secretion.}

The nasal secretion used in the following experiments was obtained either by collection direct from the nasopharynx by means of a suction pump, or by the employment of naso-pharyngeal swabs such as those used in the routine swabbing of cerebro-spinal fever contacts. This

1 If sterile dilute nasal secretion be tested against the action of sterile dilute oral saliva in stimulating the growth of the meningococcus, it will be found that oral secretion has also some power in this respect, but this is much less than with nasal secretion. Gordon has shown (Brit. Med Jour., June 17th, 1916, p. 849) that in the unsterile state oral saliva has a marked inhibiting action on the growth of this germ. When the various mouth bacteria were removed from this secretion by sterilisation or the centrifuge, this inhibiting action immediately disappeared.

2 It can be precipitated with phnsphotungstic acid and neutral lead acetate, bat this needs further confirmation.

3 In the experiments described in the present paper the medium used is Crowe's " ehocolate medium" (see THE LANCET, Nnv, 20th, 1915). In other experiments similar results have been obtained with Gordon's legumen trypagar. latter method proved the most convenient. These swabs are made of ether-washed cotton-wool placed on the ends of flexible wires which are introduced into the naso-pharyngeal region through the mouth retracted within bent glass tubes, so that all contamination from the oral secretion is avoided. The swabs were then extracted in distilled water. It was found that quite a considerable amount of secretion could be obtained in this way. This will be referred to in the following experiments as "dilute" nasal secretion in distinction to that collected "undilute" direct from the mucous membrane of the naso-pharynx, by means of the pump.

3. The Preparation of Various Extraots of Nasal Secretion.

(a) General treatment in the preparation of aqueous, alcoholic, and ethereal extracts. The cotton-wool was removed from the swab wires and covered with the solvent in glass-stoppered specimen tubes of suitable size, using 1-2 c.c. for each swab. After standing for 24 to 48 hours, the liquid was removed with a pipette, and filtered through a 5 c.c. Swedish acid-extracted filter-paper. Aqueous extracts which were further treated; and all alcoholic and ethereal extracts, were evaporated to dryness on a tepid water-bath at a temperature of about $60^{\circ} \mathrm{C}$., the basin being removed before it was absolutely dry when water was the solvent, and then taken up in distilled water, 1 c.c. of water being allowed for each swab extracted.

(b) Preparation of hydrolysed nasal secretion. 3 c.c. of undilute nasal slime was heated with ten times its volume of strong $\mathrm{HCI}$ in a test-tube standing in boiling water for six hours. Hore $\mathrm{HCl}$ was added to make up for the loss by evaporation, and the heating continued for six hours more. The solution, which was faintly yellow, was transferred to a porcelain dish and the excess of acid was evaporated off on the water-bath with the continual addition of water. When nearly all the acid had been removed the remainder was dried at a low temperature, dissolved in 7 c.c. of water, and made faintly alkaline to litmus paper with $\mathrm{N} / 10 \mathrm{NaOH}$, about 5 c.c. being necessary.

All the extracts before being sealed up in vaccine tubes were tested and their alkalinity adjusted as closely as possible to $\mathrm{Ph} .=7 \cdot 35$ of Sorensen's scale. The extracts after being sealed up were finally sterilised at $55^{\circ} \mathrm{C}$. for an hour. All manipulation of the extract was conducted in beakers of Jena glass, or Berlin porcelain or platinum, and great care was taken to have all glassware, especially the slides used for mixing the dilutions, clean and free from any chemicals. The distilled water used was redistilled from glass several times.

\section{Method of Testing the Action of Extrasts.}

The action of the various extracts of nasal secretion in stimulating the growth of the meningococcus and other bacteria was tested by making a number of dilutions of the germ in the extract, and planting uut each dilution separately and comparing their growth, with a similar quantity of the same emulsion diluted in a similar manner in distilled water or Ringer's solution, and planted out in the same way. It was found after several trials that distilled water offered the best form of control. In the following experiments care was always taken to make the control dilution first and to plant it out immediately. Thus all harmful action of distilled water on the germ was minimised as much as possible. ${ }^{\bar{j}}$ Where typhoid and coli organisms were used all emulsions and controls were made in Ringer's solution

A large number of methods of dilution were tried. The one found most useful consisted in taking up in a calibrated pipette, finely throttled to ensure accurate measurement, nine volumes of the fluid to be tested, each volume representing $25 \mathrm{c.mm}$. of liquid. They were then spread out separately on sterile slides. Then with a smaller pipette, also throttled, $5 \mathrm{c} . \mathrm{mm}$. of a standard emulsion ${ }^{6}$ of the meningococcus (5000 million to the c c.) in Ringer's solution, was drawn up. This was added to the first of the $25 \mathrm{cmm}$. volumes on the slide and thoroughly mixed by drawing it up to a fixed

4 For the proper preparation of the extracts the Medical Research Committee have kindly placed the services of Mr. D. Matthews at my disposal.

5 I have shown elsewhere (THE LANCET, 1916, ii., 902) that the meningococcus is peculiarly resistant to the hypotonic action of distilled water. I have shown that it can withstand its action for many hours without experiencing any apparent ill-effect.

"In speaking of a "standard emulsion" of the meningococcus in the following experiments it will always be understood that one in Ringer's solution of the strength of $5,000,000,000$ cocci to the cubic centimetre is meant. 